2003

\title{
Laser induced fluorescence in Ar and He plasmas with a tunable diode laser
}

\author{
R. F. Boivin \\ E.E. Scime
}

Follow this and additional works at: https://researchrepository.wvu.edu/faculty_publications

\section{Digital Commons Citation}

Boivin, R. F. and Scime, E. E., "Laser induced fluorescence in Ar and He plasmas with a tunable diode laser" (2003). Faculty Scholarship. 210.

https://researchrepository.wvu.edu/faculty_publications/210 


\title{
Laser induced fluorescence in Ar and He plasmas with a tunable diode laser
}

\author{
R. F. Boivin ${ }^{\text {a) }}$ \\ Department of Physics, Auburn University, Auburn, Alabama 36849-5311 \\ E. E. Scime \\ Department of Physics, West Virginia University, Morgantown, West Virginia 26506-6315
}

(Received 23 May 2003; accepted 30 June 2003)

\begin{abstract}
A diode laser based laser induced fluorescence (LIF) diagnostic that uses an inexpensive diode laser system is described. This LIF diagnostic has been developed on the hot helicon experiment (HELIX) plasma device. The same diode laser is used to alternatively pump Ar II and He I transitions to obtain argon ion and atomic helium temperatures, respectively. The $1.5 \mathrm{MHz}$ bandwidth diode laser has a Littrow external cavity with a mode-hop free tuning range up to $14 \mathrm{GHz}(\approx 0.021 \mathrm{~nm})$ and a total power output of about $12 \mathrm{~mW}$. Wavelength scanning is achieved by varying the voltage on a piezoelectric controlled grating located within the laser cavity. The fluorescence radiation is monitored with a photomultiplier detector. A narrow band interference filter is used to eliminate all but the plasma radiation in the immediate vicinity of the fluorescence wavelength. Lock-in amplification is used to isolate the fluorescence signal from noise and electron-impact induced radiation. For the Ar ion, the laser tuned at $668.43 \mathrm{~nm}$ is used to pump the $3 d^{4} F_{7 / 2} \mathrm{Ar}$ II metastable level to the $4 p^{4} D_{5 / 2}$ level. The $442.60 \mathrm{~nm}$ fluorescence radiation between the $4 p^{4} D_{5 / 2}$ and the $4 s^{4} P_{3 / 2}$ levels is captured by the photomultiplier tube. For atomic He, the laser is tuned at 667.82 $\mathrm{nm}$ to pump a fraction of the electron population from the $2^{1} \mathrm{P}$ state to the $3{ }^{1} \mathrm{D}$ upper level. Although the $2^{1} P$ level is not a metastable, the close proximity of $2^{1} S$ metastable makes this new He I LIF scheme possible. In this scheme, a fraction of the laser-excited electrons undergo collisional excitation transfer from the $3{ }^{1} D$ to the $3{ }^{1} P$ level. In turn, the $3{ }^{1} P$ state decays to the metastable $2^{1} S$ by emitting $501.57 \mathrm{~nm}$ fluorescence photons. (C) 2003 American Institute of
\end{abstract} Physics. [DOI: 10.1063/1.1606095]

\section{INTRODUCTION}

Laser induced fluorescence (LIF) was first introduced to measure the ion velocity distribution function (ivdf) in an argon plasma over 25 years ago. ${ }^{1}$ Since then, LIF using high power, single mode, tunable dye lasers has been employed in many types of plasma discharges to measure ion flow, ion temperature, magnetic field strength, and plasma density. ${ }^{2-10}$ Recently, LIF with tunable dye lasers was used to measure both ion and neutral temperatures in high density, low temperature, helicon plasmas. ${ }^{11-14}$ Typically, for LIF in argon plasmas with a dye laser; a doublet transition scheme is routinely used. In this scheme, the Ar II $3 d^{2} G_{9 / 2}$ metastable state is pumped to the $4 p^{2} F_{7 / 2}$ excited level by a laser tuned to $611.49 \mathrm{~nm}$. The $4 p^{2} F_{7 / 2}$ excited state decays to the $4 s^{2} D_{5 / 2}$ state by emitting $460.96 \mathrm{~nm}$ photons. Unfortunately, the $600-630 \mathrm{~nm}$ spectral range is presently inaccessible by commercial diode lasers. However, the Ar ion has many other metastable states that can be used for LIF measurements. Two diode laser accessible LIF schemes already tested in low temperature argon plasmas utilize quartet state metastables as initial states. ${ }^{15,16}$ The two pump lines are part of the intense ${ }^{4} F \rightarrow{ }^{4} D$ quartet transition array. ${ }^{17}$ Since these quartet metastable states have lower energy levels than the

${ }^{a)}$ Electronic mail: boivin@physics.auburn.edu
$3 d^{2} G_{9 / 2}$ doublet metastable state, they are likely more populated than the doublet metastable state in low temperature plasmas. ${ }^{18}$ Thus for the same amount of incident laser power and since the transition probabilities are similar, the LIF intensity of the quartet scheme should be larger than the standard doublet transition scheme.

Excellent reviews covering the different aspects of diode laser systems are given in Refs. 19 and 20. Diode lasers have a number of advantages over dye lasers: lower cost, easier maintenance, nontoxicity, better day-to-day stability, and considerably smaller size. ${ }^{19,20}$ On the other hand, diode laser use has been limited by a number of technological issues: lack of available emitting diodes at desired wavelengths, diode lifetimes, and low output power in the visible and in the near infrared range. Individual diode lasers are also quite limited in terms of tuning range, typically $10-15 \mathrm{~nm}$. This is much smaller than the average $100 \mathrm{~nm}$ tuning range associated with each dye-optics combination for dye laser systems.

Over the past decade, technological improvements have extended diode lifetimes and increased the range of available wavelengths to the point where diode lasers can be used for LIF measurements. Two different approaches are possible. The first method makes use of tapered laser amplifiers in conjunction with a low power seed diode laser. ${ }^{15}$ In this approach, the output of the tuned seed laser $(<5 \mathrm{~mW})$ is amplified through a master oscillator power amplifier (MOPA) 


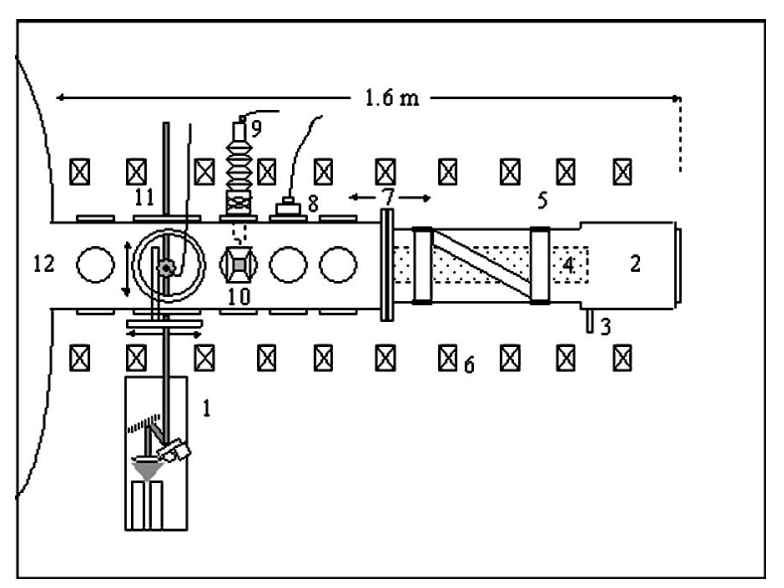

FIG. 1. Upper view of the HELIX plasma source. 1: Diode laser, 2: pumping station, 3: gas inlet, 4: plasma column, 5: fractional helix antenna, 6: magnetic field coils, 7: glass chamber section (right), S.S. chamber section (left), 8: spectroscopy optics, 9: retractable rf compensated Langmuir probe, 10: microwave interferometer, 11: 2D LIF collection optics, and 12: large space chamber LEIA.

generating a $100 \mathrm{~mW}$ tunable laser output. Although these MOPA amplifiers are currently commercially available for specific wavelengths in the infrared range (fiber-optic signal booster for telecommunication systems), the product line that covered the red-visible range was discontinued because of reliability and profitability issues.

In this work, we show that a second approach using a single, low-power diode laser system is possible. We present LIF measurements of argon ions accomplished with an unamplified diode laser in a Littrow external cavity. Furthermore, we also show that, by a judicious choice of transitions, the same diode laser can be used to measure the velocity distribution function of neutral helium atoms in low temperature plasmas. While the LIF scheme used for Ar II has been used previously, ${ }^{15}$ the He I scheme presented in this work is original. To the best of our knowledge, this work is the first report of LIF measurements with a simple unamplified, Littrow cavity, diode laser system in low temperature plasmas. Our LIF diagnostic has also successfully measured argon ion temperatures and ion drifts in the magnetic nozzle experiment (MNX) device at Princeton Plasma Physics Laboratory (PPPL). ${ }^{21}$

\section{EXPERIMENTAL APPARATUS}

\section{A. Helicon plasma source (HELIX)}

The experiments reported here were performed in the hot helicon experiment (HELIX) helicon plasma source. The HELIX (Fig. 1) vacuum chamber is composed of two distinct sections: a $0.60 \mathrm{~m}$ long, $0.1 \mathrm{~m}$ diameter Pyrex tube where the copper antenna wraps around and couples rf radiation into the plasma and a $0.91 \mathrm{~m}$ long, 0.15 diameter stainless steel section with ports distributed along its length. The stainless steel chamber has one set of four 6 in. Conflat ${ }^{\mathrm{TM}}$ crossing ports in the center of the chamber that are used for LIF measurements. The opposite end of the stainless steel chamber opens into a $4.5 \mathrm{~m}$ long, $2 \mathrm{~m}$ diameter space simulation chamber, LEIA. ${ }^{12}$ Three turbomolecular drag pumps with a total pumping speed of $3740 \mathrm{l} / \mathrm{s}$ are connected to the vacuum chamber. The base pressure in the system is 1 $\times 10^{-7}$ Torr. Any gas or gas mixture can be injected through a precision MKS mass flow controller ${ }^{22}$ mounted in a flange at the end of the small chamber. Operating gas pressures range from 0.1 to more than 100 mTorr. Ten electromagnets produce a steady state axial magnetic field of $0-1300 \mathrm{G}^{23}$ An rf amplifier supplies up to $2 \mathrm{~kW}$ of rf power to the plasma through a standard $\pi$ matching circuit over the $6-18 \mathrm{MHz}$ frequency range. The injected power is limited by the quality of the match. A $19 \mathrm{~cm}$ long, $m=+1$, fractional helical antenna ${ }^{24}$ couples the rf energy into the plasma. Typical electron temperatures and densities in HELIX are $T_{e} \approx 4 \mathrm{eV}$ and $n \geqslant 1 \times 10^{13} \mathrm{~cm}^{3}$ for argon and $T_{e} \approx 8 \mathrm{eV}$ and $n \geqslant 1$ $\times 10^{12} \mathrm{~cm}^{3}$ for helium as measured with an rf compensated Langmuir probe. ${ }^{25}$

\section{B. Diode laser}

A diode laser can be described as a complex "sandwich" of semiconductor material. Laser light is generated by sending a current through the active region of the diode located between the $n$ - and $p$-type cladding layers. ${ }^{19}$ The injected current produces electrons and holes, which in turn recombine and emit photons. A narrow channel in the active region defines the spatial mode of the laser. The rectangular shape of the gain region creates an elliptic radiation pattern (typical aspect ratio 3:1). The laser output is typically broadband $(\mathrm{GHz})$ and highly polarized (linear with a 100:1 ratio). The laser wavelength is determined by the band gap of the semiconductor material. ${ }^{20}$ An external cavity is used to reduce the laser bandwidth ( $\mathrm{GHz}$ to $\mathrm{MHz}$ ) and to fine tune the laser to the desired wavelength. Two possible cavity configurations are currently available: Littrow or Littman. ${ }^{26,27}$ In the Littrow configuration, tuning is achieved by changing the angle of the beam with respect to the grating normal. The Littrow cavity offers more output power at the expense of tuning range. In the Littman configuration, tuning is achieved by tilting the tuning mirror, thereby changing the refracted wavelength fed back into the cavity. The Littman cavity is a double pass grazing incidence cavity that naturally achieves multimode suppression. This configuration has typically larger mode-hop free tuning range $(10 \times$ Littrow) but at the expense of output power $(50 \%-70 \%$ of a comparable Littrow laser because of the loss of the zeroth order of the reflected beam). ${ }^{27}$ Because of the crucial importance of laser power for LIF measurements we choose a Littrow cavity diode laser. The diode laser cavity configuration is shown in Fig. 1.

The tunable diode laser used in these experiments is a Sacher Lasertechnik ${ }^{28}$ model SAL-665-10 with an external Littrow cavity comprised of a piezoelectric transducer controlled (PZT) grating with a beam correction mirror. This Peltier cooled diode laser is characterized by a $1.5 \mathrm{MHz}$ bandwidth and a $12 \mathrm{~mW}$ power output. The diode laser has a coarse tuning range of approximately $12 \mathrm{~nm}$ around the factory set central wavelength $\left(668 \pm 6 \mathrm{~nm}\right.$ for our laser) ${ }^{28}$ Coarse tuning is accomplished by mechanically adjusting the cavity length with a screwdriver. While coarse tuning, the wavelength jumps by discrete values within the tuning range. 


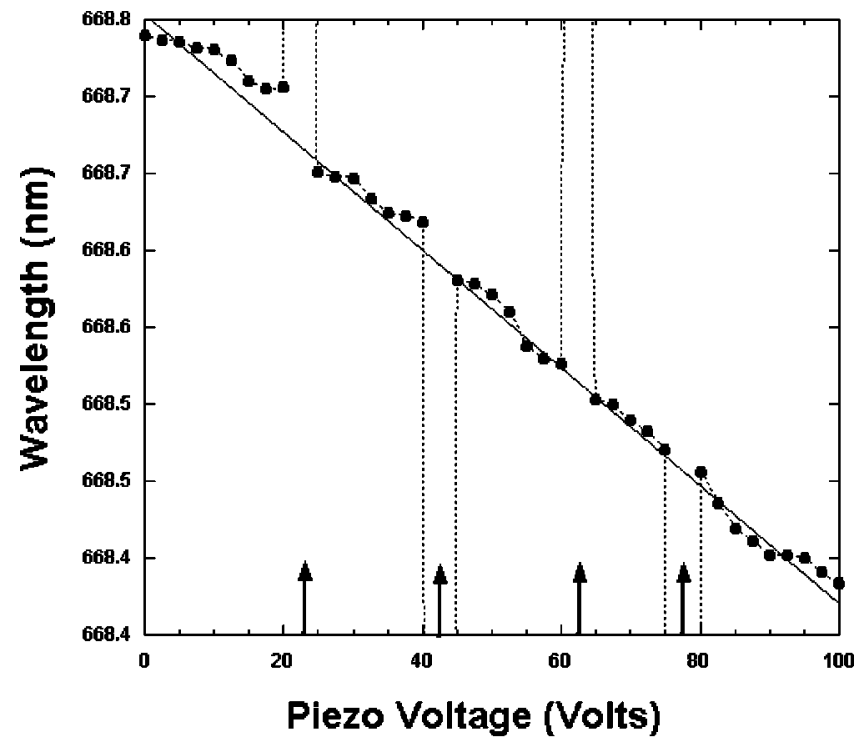

(a)

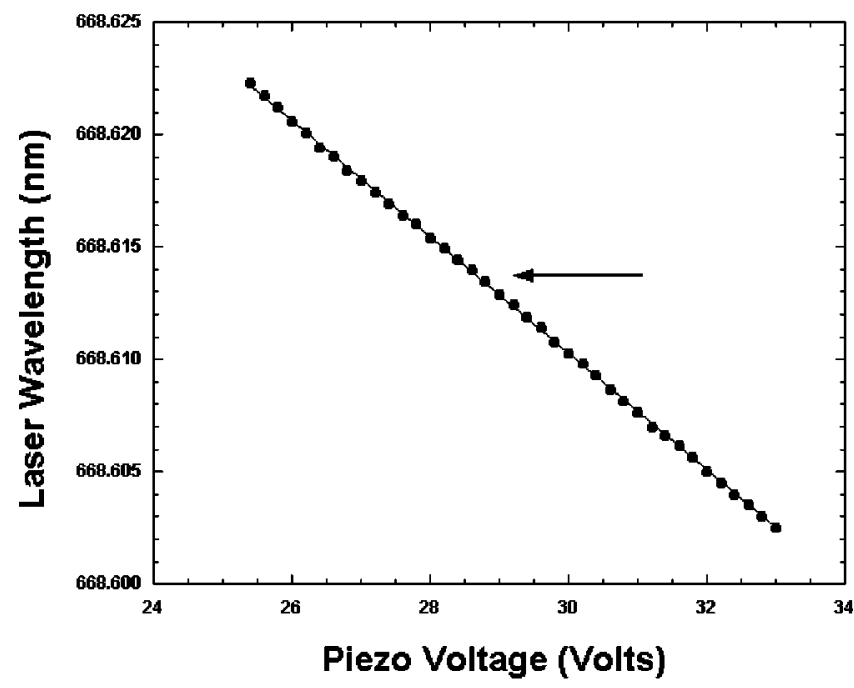

(b)

FIG. 2. (a) Fine-tuning range obtained by varying the voltage on the piezoelectric actuator. Mode-hops are indicated by arrows. Mode-hop free regions within the fine-tuning range are clearly visible. (b) Mode hop free region (14 $\mathrm{GHz}$ ) centered on the Ar II pump transition (668.614 nm in vacuum, indicated by arrow). The temperature of the laser is slightly different than in (a). The wavelength is precisely measured for every voltage increment.

The SAL-665-10 laser has also a fine tuning range of 0.4 $\mathrm{nm}$ (or $300 \mathrm{GHz}$ ). Fine tuning is accomplished by either changing the voltage on the piezoelectric (PZT) actuator or by changing the temperature of the cavity. Both procedures effectively modify the length of the cavity, thereby changing the output wavelength. Single mode tuning without mode hops is achieved when the external cavity modes and the dispersion bandwidth shift in unison so that the same mode lases at all wavelengths throughout the tuning range. A mode-hop occurs when the external cavity modes and the dispersion curve are shifted relative to each other by exactly one external cavity mode [see Fig. 2(a)]. At this point, the adjacent mode becomes more centered in the gain profile and the wavelength jumps discontinuously to the adjacent mode. The cavity length and the incident angle (laser grating angle) are coupled in the tuning process and must be controlled simultaneously.

The need for accurate measurements of the absorption linewidth in plasmas by LIF places a number of constraints on the laser. First, the laser bandwidth should be smaller than the linewidth of the probed species. In our experiments, the laser bandwidth of $\sim 1.5 \mathrm{MHz}$ is much smaller than the 2 and $3 \mathrm{GHz}$ full width at half maximum (FWHM) expected for $0.3 \mathrm{eV}$ Ar II and $0.07 \mathrm{eV}$ He I transitions, respectively (see Sec. III). Second, no mode-hops can occur over the line absorption spectrum. The laser wavelength must smoothly change while sweeping the entire absorption spectrum to produce the desired fluorescence. Using a Burleigh wavemeter, ${ }^{29}$ the wavelength of the Littrow cavity diode laser is monitored as a function of the piezoelectric voltage and shown in Fig. 2(a). The wavelength variation as a function of cavity temperature shows a similar behavior. The full $300 \mathrm{GHz}$ fine tuning range is plagued by a number of modehops. The mode-hops limit the wavelength interval that can be used to obtain the absorption line shape. However, as can be seen in Fig. 2(a), many smaller mode-hop free tuning regions are available within the fine tuning range. Furthermore, since the pattern of mode-hops remains roughly the same within the fine tuning range and for the lifetime of the diode, the best mode-hop free region is easily recognizable and can be selected in a matter of minutes. In our experiments, we choose to ramp the voltage on the PZT actuator to vary the wavelength and set the central wavelength by adjusting the cavity temperature. ${ }^{15}$ The Sacher LaserTechnik MLD-1000 laser controller ${ }^{28}$ in conjunction with a National Instruments I/O card ${ }^{30}$ is used to sweep the PZT voltage. A precise set of wavelength measurements was performed within a tuning region without mode-hops and is shown in Fig. 2(b). The chosen $14 \mathrm{GHz}$ wide mode-hop free region is sufficient to cover the entire argon ivdf and is centered on the central frequency for the Ar II pump transition, namely $668.430 \mathrm{~nm}(668.614 \mathrm{~nm}$ in vacuum). For He I, the pattern of mode-hop free regions is slightly different since the output wavelength must be tuned to $667.82 \mathrm{~nm}$. However, many 14 $\mathrm{GHz}$ wide mode-hop free regions are available within the fine tuning range and by slightly changing the cavity temperature we were able to center a mode-hop free region on the absorption wavelength.

The third constraint on the laser system is that the laser power must remain constant over the absorption line. Using an Oriel laser powermeter, ${ }^{31}$ laser power fluctuations of less than 5\% are observed over the entire fine tuning range (300 $\mathrm{GHz}$ ). Within a single mode-hop free tuning region the fluctuations are even smaller $(<3 \%)$. The measured power fluctuations are only slightly larger than the stated precision of the powermeter. ${ }^{31}$ Furthermore, since the expected line will occupy only the central part of the fluorescence spectrum (about 4 of the $14 \mathrm{GHz}$ ); the laser power only needs to be constant near the center of the mode-hop free region. Similar laser power fluctuations are observed near the He I central absorption wavelength.

Finally, the diode laser wavelength must not drift during the LIF measurement. LIF measurements with a drifting laser will be characterized by a nonreproducible asymmetric 


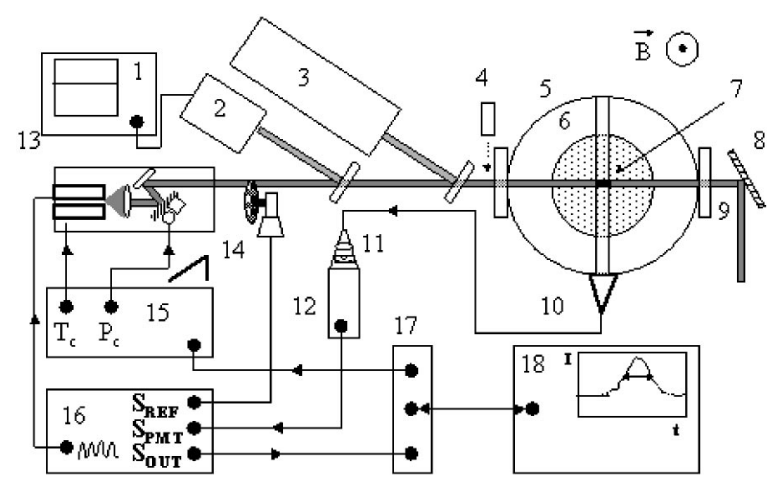

FIG. 3. LIF experimental setup. 1: scope, 2: powermeter, 3: wavemeter, 4: $\frac{1}{4}$-wave retarder/polarizer, 5: vacuum chamber, 6: plasma, 7: LIF volume, 8: laser dump, 9: collection optics, 10: interference filter, 11: PMT, 12: laser, 13: chopper, 14: laser controller, 15: lock-in amplifier, 16: acquisition board, 17: computer, and 18: fluorescence signal.

distribution instead of the Gaussian distribution expected for Doppler broadening (see Sec. III A). We found that the best way to achieve stable laser operation was to keep the temperature constant around the laser, to frequently monitor the wavelength with a wavemeter, and to minimize the LIF acquisition time. Measurements taking up to $10 \mathrm{~min}$ are possible without significant drift, although the measurements featured in this article were all completed within $3 \mathrm{~min}$. We found that it also helps to let the laser "warm up" for an hour or so before any measurements are made. The diode laser current must also remain constant during the LIF measurements.

\section{LIF apparatus}

A schematic of the entire LIF system is shown in Fig. 3. A photodiode connected to an oscilloscope is used to monitor the laser output for mode-hop detection (a mode-hop is characterized by a sudden change in laser power output and can easily be observed on an oscilloscope). The wavemeter is used to ensure that the mode-hop free region stays centered on the absorption line. The entire laser can be rotated $90^{\circ}$ to choose the appropriate linear polarization (relative to the magnetic field direction) or the $\frac{1}{4}$-wave plate retarder can be used to convert the linearly polarized light into circularly polarized light. The LIF sampling volume is formed by the intersection of the irradiated volume and the detection volume within the plasma column. A beam dump is used to prevent reflected laser light from coming backing into the vacuum chamber.

Light detection is achieved by means of a set of collection optics to a fiber optic cable. Since the fluorescence radiation is essentially unpolarized (a mix of linearly and circularly polarized light), no information is lost by using multimode fiber optics in the collection optics. The light collected by the fiber optics is then filtered by a $1 \mathrm{~nm}$ bandwidth interference filter ${ }^{32}$ and sent into a narrow-band, high-gain, Hamamatsu photomultiplier tube (PMT). ${ }^{33}$ The PMT signal is composed of fluorescence radiation, electron impact induced radiation, and electronic noise. A $4 \mathrm{kHz}$ mechanical chopper is used to modulate the laser beam before it enters the vacuum chamber and a Stanford Research SR830 lock-in amplifier $^{34}$ is used to eliminate all noncorrelated signals. Lock-in amplification is indispensable since the electronimpact induced emission is several orders of magnitude larger than the fluorescence signal. The electronically filtered LIF signal (and correlated noise) is sent to the computer via the acquisition board for data analysis.

Estimating the error in an individual temperature measurement is nontrivial. The temperature uncertainty depends on: the signal-to-noise $(\mathrm{S} / \mathrm{N})$ ratio of the measurement, the finite laser bandwidth, laser wavelength drift during the measurement, fluctuation in the laser power output over the absorption line, plasma fluctuations within the column, and variation of the magnetic field strength within the measurement volume. For S/N ratios larger than 20, we estimate the temperature error to be less than $5 \%$. The uncertainty doubles when the $\mathrm{S} / \mathrm{N}$ ratio decreases by a factor of 2 . This overall precision was confirmed during LIF measurements of neutral helium in high pressure, low power discharges (under these conditions neutral helium is essentially at room temperature, see Sec. III B).

\section{LASER INDUCED FLUORESCENCE}

In a typical LIF measurement, the frequency of a very narrow bandwidth laser is swept across a collection of ions or atoms that have a thermally broadened velocity distribution. The atoms or ions absorb a photon when it is at the appropriate frequency in its rest frame. After a short time, depending on the lifetime of the excited state, the atom or ion emits a photon of either the same frequency or another frequency. Measurement of intensity of the emitted photons as a function of laser frequency consitutes a LIF measurement. The shift in the center frequency and the width of the absorption spectrum feature for the entire ensemble of atoms or ions is then used to determine the flow velocity and temperature of the particle distribution. Optimally, a three level system involving an absorption line different from the fluorescence emission is used to reduce the effects of scattered light and background emission at the pump wavelength.

\section{A. Ar II LIF}

First proposed by Severn et al., ${ }^{15}$ the three-level Ar II scheme used in these experiments makes use of a quartet metastable state to initiate the LIF process. In this scheme, the laser, tuned at $668.43 \mathrm{~nm}$, is used to pump the $3 d^{4} F_{7 / 2}$ Ar II metastable level to the $4 p{ }^{4} D_{5 / 2}$ level. A fraction of the $442.60 \mathrm{~nm}$ fluorescence radiation between the $4 p{ }^{4} D_{5 / 2}$ and the $4{ }^{4} P_{3 / 2}$ levels is collected by the PMT, yielding the absorption line shape. Since the energy associated with the quartet $3 d^{4} F_{7 / 2}$ metastable level $(17.69 \mathrm{eV})$ is lower than the energy of the $3 d^{2} G_{9 / 2}$ doublet metastable $(19.12 \mathrm{eV}),{ }^{18}$ the quartet level is likely to be more populated than the doublet metastable in low temperature plasmas ${ }^{35}$ and since the transition probabilities are nearly equal, ${ }^{36}$ the LIF intensity of the quartet scheme should be larger for the same incident laser power.

As with any spectroscopic measurement of a magnetized plasma, the measured line broadening includes contributions from many different mechanisms. In the present LIF con- 
TABLE I. Approximate linewidths associated with different line broadening mechanisms. The Doppler broadening arises from the thermal motion of ions. The tabulated average Zeeman splitting is the average shift of the $\pi$ cluster with respect to the unshifted transition for $B=1 \mathrm{kG}$. The calculated power broadening is for line saturation effects due to the incident laser power density, the natural linewidth is due to the Heisenberg uncertainty principle for the absorption transition, and the instrumental broadening is the finite laser bandwidth.

\begin{tabular}{ll}
\hline \hline \multicolumn{1}{c}{ Broadening mechanism } & FWHM value $(\mathrm{nm})$ \\
\hline Doppler broadening & $\sim 6.4 \times 10^{-3}$ \\
Average Zeeman splitting $(\pi$ cluster $)$ & $\sim 3.5 \times 10^{-3}$ \\
Power broadening & $\sim 1.0 \times 10^{-5}$ \\
Natural linewidth & $\sim 3.1 \times 10^{-7}$ \\
Pressure broadening & $\sim 1.2 \times 10^{-6}$ \\
Instrumental broadening & $\sim 1.3 \times 10^{-6}$ \\
\hline \hline
\end{tabular}

figuration: natural broadening, Doppler broadening, Zeeman broadening, pressure or Stark broadening, and power broadening could all contribute to the measured absorption line shape. ${ }^{37-46}$ For the argon ion LIF scheme in a typical helicon source $\left(B=1000 \mathrm{G}, \quad n_{e}=10^{13} \mathrm{~cm}^{-3}, \quad T_{i}=0.1 \mathrm{eV}, \quad\right.$ and pressure $=4 \mathrm{mTorr})$ and a diode laser system $(\Delta \nu$ $\approx 1.5 \mathrm{MHz}$ ), the magnitudes of the different line broadening mechanisms are listed in Table I. Except for Zeeman splitting at high magnetic field strengths, Doppler broadening clearly dominates the absorption line shape. For the case where broadening depends solely on the thermal motion, the resulting linewidth can be written in terms as

$$
I_{R}(\nu)=I_{R}\left(\nu_{o}\right) \exp \left[\frac{-m_{i} c^{2}\left(\nu-\nu_{o}\right)^{2}}{2 k T_{i} \nu_{o}^{2}}\right],
$$

where $I_{R}\left(\nu_{o}\right)$ is the maximum intensity, $\nu_{o}$ is the unshifted central frequency, and $T_{i}, m_{i}$ are the temperature and mass of the ion (or neutral), respectively. The Doppler-broadened full width at half maximum (FWHM) is $\Delta \nu_{D}$ $=\left(8 \ln 2 k T_{i} \nu_{o}^{2} m_{i}^{-1} c^{-2}\right)^{1 / 2}$. Defining the $\alpha_{D}$ as the Doppler fitting parameter, $\left(\alpha_{D}=2 k \nu_{o}^{2} m_{i}^{-1} c^{-2}\right)$, Eq. (1) can be written as

$$
I_{R}(\nu)=I_{R}\left(\nu_{o}\right) \exp \left[\frac{-\left(\nu-\nu_{o}\right)^{2}}{\alpha_{D} T_{i}}\right] .
$$

In this form, only $\alpha_{D}$ needs to be changed for a different pump transition for a given species. For the Ar II $(668.43 \mathrm{~nm}$ transition), $\left(\alpha_{D}\right)^{-1}=0.092495 \mathrm{eV}(\mathrm{GHz})^{-2}$ and, for He I $(667.82 \mathrm{~nm}),\left(\alpha_{D}\right)^{-1}=0.009311 \mathrm{eV}(\mathrm{GHz})^{-2}$.

For typical helicon plasma source experiments, the total absorption line shape will be a convolution of Doppler broadening and Zeeman splitting, i.e., each component of the observed Zeeman splitting is broadened by thermal motion. For the specific Ar II quartet transition used in this work, the Zeeman splitting is composed of three separated line clusters containing a total of 18 transitions. ${ }^{44}$ Due to the symmetry of the Zeeman pattern, only the Zeeman splitting for one side of the central wavelength needs to be calculated. For the six $\pi$ transitions, the magnetic orbital quantum number for each level is the same $(\Delta M=0)$; the calculated values for a $1 \mathrm{kG}$ magnetic field are shown in Table II. For the $12 \sigma$ transitions (six for each cluster), the magnetic orbital quantum number for each level is different $(\Delta M= \pm 1)$; the calculated values
TABLE II. Zeeman splittings for the $3 d^{4} F_{7 / 2}$ Ar II level to $4 p{ }^{4} D_{5 / 2}$ transitions $(B=1 \mathrm{kG})$. First and second columns: magnetic orbital quantum number of first $\left(M_{i}\right)$ and second LIF level $\left(M_{f}\right)$, respectively. Third column: shift of the Zeeman component with respect to the unshifted central line. Fourth column: relative weight of the different Zeeman components according to the Wigner-Eckart theorem. The $\pi$ transitions correspond to the condition $\left(M_{i}=M_{f}\right)$. The $\sigma$ transitions correspond to the condition $\left(M_{i} \neq M_{f}\right)$.

\begin{tabular}{cccc}
\hline \hline$M_{i}$ & $M_{f}$ & $\Delta \lambda(\AA)$ & $w$ \\
\hline $1 / 2$ & $1 / 2$ & $1.38 \times 10^{-3}$ & 0.1071 \\
$3 / 2$ & $3 / 2$ & $4.16 \times 10^{-3}$ & 0.0892 \\
$5 / 2$ & $5 / 2$ & $6.93 \times 10^{-3}$ & 0.0536 \\
$7 / 2$ & $5 / 2$ & $1.88 \times 10^{-2}$ & 0.09375 \\
$5 / 2$ & $3 / 2$ & $2.15 \times 10^{-2}$ & 0.06696 \\
$3 / 2$ & $1 / 2$ & $2.43 \times 10^{-2}$ & 0.04464 \\
$1 / 2$ & $-1 / 2$ & $2.71 \times 10^{-2}$ & 0.02679 \\
$-1 / 2$ & $-3 / 2$ & $2.99 \times 10^{-2}$ & 0.01339 \\
$-3 / 2$ & $-5 / 2$ & $3.27 \times 10^{-2}$ & 0.00446 \\
\hline \hline
\end{tabular}

for a $1 \mathrm{kG}$ magnetic field are shown in Table II. The relative intensities of each Zeeman component can be evaluated using the Wigner-Eckart theorem ${ }^{44,47}$ and are also shown in Table II. The line distribution of the $\pi$ cluster is symmetric around the central wavelength while the two $\sigma$ line clusters are asymmetric with their most intense lines closest to the central wavelength.

The polarization of the laser can be adjusted in such a fashion that only one of three clusters will be excited. When the polarization of the laser is parallel to the axial magnetic field, only the $\pi$ cluster is pumped. When the polarization is perpendicular to the axial magnetic field, then the two $\sigma$ clusters are pumped. Adding a quarter-wavelength retarder converts the linearly polarized laser light into circularly polarized light and then only the $\sigma$ cluster with the same polarization is pumped. For the single cluster configurations listed above, the absorption line shape can be best described as the envelope (convolution) that encompasses all the different Doppler broadened Zeeman components. The resulting intensity function is a series of superimposed Gaussian distributions of different intensities each located at a different frequency shift with respect to the central frequency. The resulting intensity function $I_{R}(\nu)$ for the $\pi$ cluster can be written as ${ }^{47}$

$$
I_{R}(\nu)=\sum_{i=1}^{n} I_{i}\left(\nu_{o}-\Delta \nu_{i}\right) \exp \left[\frac{-\left(\nu-\Delta \nu_{i}-\nu_{o}\right)^{2}}{\alpha_{D} T_{i}}\right],
$$

where $\Delta \nu_{i}$ and $I_{i}\left(\nu_{o}-\Delta \nu_{i}\right)$ are the spacings relative to the central frequency $\nu_{o}$ and the relative intensities of the $n=6$ different Zeeman components, respectively. The $\Delta \nu_{i}$ 's can be written in terms of the central frequency and the magnetic field strength: $\Delta \nu_{i}= \pm \varepsilon_{i} \nu_{o} B$, where the $\varepsilon_{i}$ are shift coefficients for each Zeeman component with respect to the central frequency. The + and - signs indicate that the Zeeman components are located on both sides of the central frequency. Similar, but asymmetric expressions, can be written for each of the $\sigma$ clusters. $^{47}$ For $B=0$, Eq. (3) reduces to Eq. (2), the pure Doppler broadened line shape.

A typical Ar II LIF measurement is shown in Fig. 4. The laser polarization was oriented to pump the $\pi$ cluster. The 


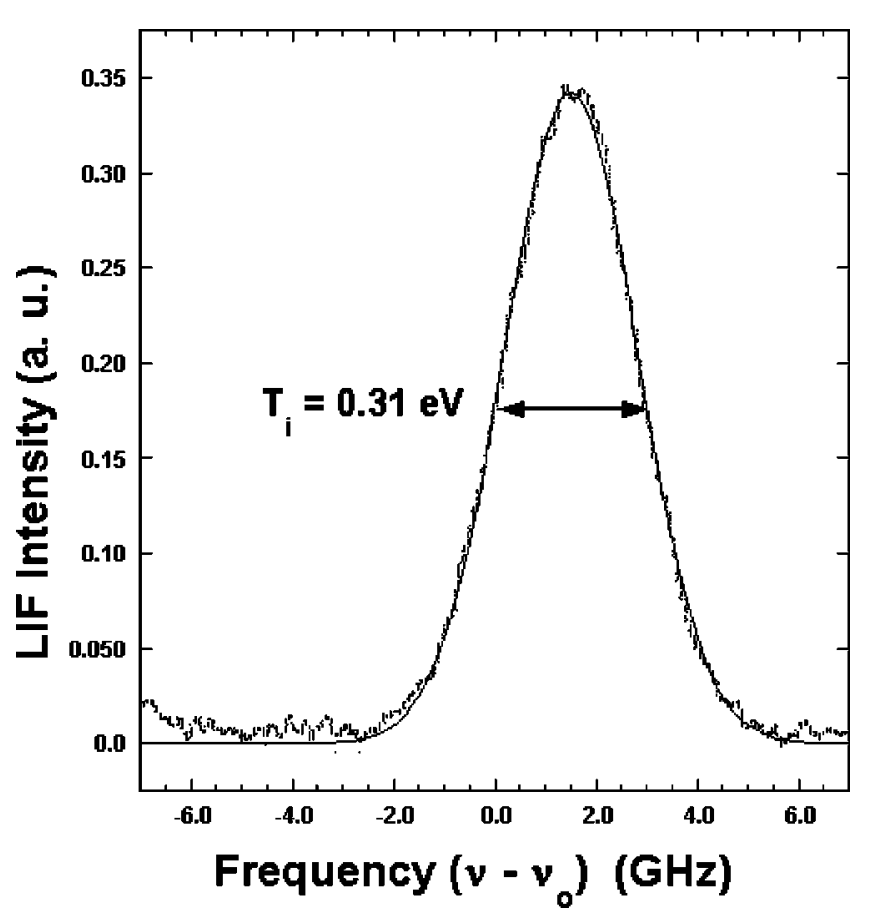

FIG. 4. Typical Ar II LIF signal vs laser frequency. The solid curve is obtained by using the deconvolution formula, Eq. (3). The optimum fit yields a $0.31 \mathrm{eV}$ ion temperature.

helicon plasma discharge was operated at $500 \mathrm{~W}, 1 \mathrm{kG}, 10$ $\mathrm{MHz}$, and $1.25 \mathrm{mTorr}$ for $\mathrm{rf}$ power, magnetic field intensity, rf frequency, and argon neutral pressure, respectively. Amazingly, the $\mathrm{S} / \mathrm{N}$ ratio (70:1) is nearly as good as obtained with a $200 \mathrm{~mW}$ dye laser system using the same collection optics with, however, a different interference filter. The remarkable $\mathrm{S} / \mathrm{N}$ ratio is due to the large population of the $3 d^{4} F_{7 / 2}$ quartet metastable in the argon plasma. Using Eq. (3), the ion temperature of the ivdf shown in Fig. 4 is $0.31 \mathrm{eV}$, consistent with dye laser LIF measurements of similar plasmas. ${ }^{13}$

In Fig. 5, we show the Ar ion temperature as a function of the neutral pressure. All the plasma parameters except the

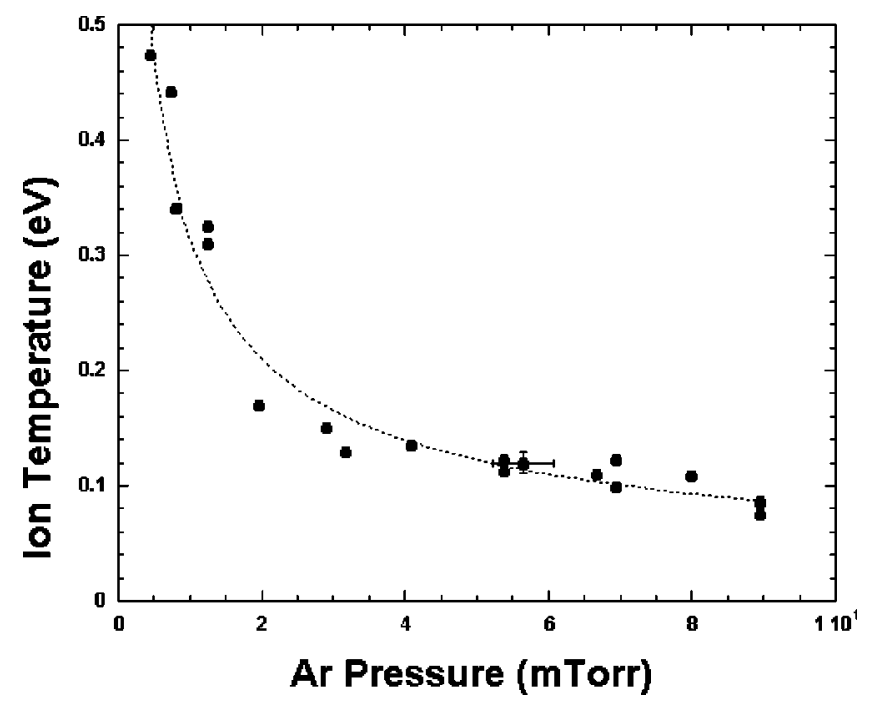

FIG. 5. Ion temperature as a function of Ar pressure. Ion temperatures are extracted by using the deconvolution formula, Eq. (3). The power function fit is only a guide to the eye.



FIG. 6. Partial Grotrian diagram of the helium atom for the two LIF schemes. The dotted lines are the dye laser LIF scheme (triplet side, right) and the hash lines the new diode laser LIF scheme (singlet side, left). Transition wavelengths are in $\mathrm{nm}$. $\mathrm{G}$ and $\mathrm{M}$ are for ground and metastable states, respectively.

pressure are the same as in Fig. 4. Similar scalings of ion temperature with neutral pressure were also observed in dye laser LIF measurements. Although Fig. 5 demonstrates that diode laser LIF measurements are possible over a substantial range of neutral pressures, the data also highlight the limitations imposed by $\mathrm{S} / \mathrm{N}$ ratio requirements. For pressures lower than $0.4 \mathrm{mTorr}$, the $\mathrm{S} / \mathrm{N}$ ratio is insufficient to obtain an accurate LIF measurement. A combination of a small metastable ion population and insufficient diode laser power is responsible for the poor $\mathrm{S} / \mathrm{N}$ ratio. At higher pressures, above 9 mTorr, the background electron-impact radiation is so intense that the lock-in amplification is incabable of isolating the LIF from the background light. In both cases, the measurements could be improved with additional diode laser power. At high neutral pressures, the diagnostic range could be extended by using more aggressive noise reduction techniques or by reducing the solid angle subtended by the collection optics. Power limitation issues may soon be a thing of the past as higher power diode lasers are now coming to market. ${ }^{28}$ These new diode lasers have approximately twice the output power of the diode laser used in this work.

\section{B. He I LIF}

Using the same diode laser at a slightly different wavelength, it is possible to measure the neutral temperature of helium plasmas. The LIF scheme used for neutral helium is an indirect scheme that involves four different energy levels of the helium atom instead of three like in the Ar II case. In a LIF scheme system, an excitation transfer process between excited levels (electron-induced collisional excitation transfer) is necessary. In many aspects, the He I diode LIF scheme used here is similar to the He I dye laser LIF scheme used earlier. ${ }^{48}$ In that scheme, shown in Fig. 6 , the $2^{3} P$ state was pumped to the $3^{3} D$ level by a dye laser tuned to $587.6 \mathrm{~nm}$. A fraction of the excited electrons in the $3^{3} D$ level then underwent either a collisional transfer excitation or an allowed IR transition $(18620 \mathrm{~nm})^{49}$ to the neighboring $3^{3} P$ level where they decayed to the $2^{3} S$ metastable state by emitting 388.9 




FIG. 7. Typical He I LIF signal vs laser frequency. The temperature is obtained by using the simple Doppler broadening function, Eq. (2). The optimum fit yields a $0.027 \mathrm{eV}$ temperature.

$\mathrm{nm}$ photons. The $3^{3} \mathrm{P}$ to $2^{3} S$ transition has a branching ratio of unity.

The diode laser LIF scheme makes use of the singlet side of the Grotrian diagram (see Fig. 6). The diode laser is tuned to $667.82 \mathrm{~nm}$ to pump electrons from the $2{ }^{1} P$ state to the $3^{1} D$ state. A fraction of the excited electrons then undergo collisional excitation transfer from the $3{ }^{1} D$ to the $3{ }^{1} P$ level. In turn, the $3^{1} P$ state decays to the metastable $2{ }^{1} S$ by emitting $501.6 \mathrm{~nm}$ photons with a branching ratio of $0.023 .{ }^{36} \mathrm{In}$ both the diode and dye laser LIF schemes, the $2^{1} P$ and $2^{3} P$ initial states are not metastable. However, large electron populations in nearby $2^{1} S$ and $2{ }^{3} S$ metastable states makes these LIF schemes possible. ${ }^{50}$ Neither of these LIF schemes are likely to work well in collisionless helium plasmas $\left(n_{e}\right.$ $<10^{10} \mathrm{~cm}^{-3}$ ) as electron-induced collisions populate the metastable states and ultimately the $2^{1} P$ and $2^{3} P$ levels.

Typical He I LIF measurements are shown in Fig. 7. Again, the laser polarization was oriented to pump the $\pi$ cluster. For singlet He I transitions, the Zeeman splitting is trivial (two symmetrically shifted $\sigma$ components and one unshifted $\pi$ component ${ }^{47}$ ) and the linewidth of the sole $\pi$ component is given by Eq. (1). The helicon plasma discharge was operated at $700 \mathrm{~W}, 600 \mathrm{G}, 11 \mathrm{MHz}$, and 9.5 mTorr for rf power, magnetic field intensity, rf frequency, and He neutral pressure, respectively. In this case the $\mathrm{S} / \mathrm{N}$ ratio, approximately 30:1, although excellent, is inferior to what can be obtained using the dye laser scheme. In addition to the smaller diode laser power, a number of other issues contribute to the reduced $\mathrm{S} / \mathrm{N}$ ratio. First and foremost, the triplet states are on average more populated than the singlet levels for the He helicon plasmas. ${ }^{50,51}$ For most states, a 3 to 1 ratio is observed except for the metastable states ${ }^{50-53}$ where the population ratio grows to about 100 when the plasma density

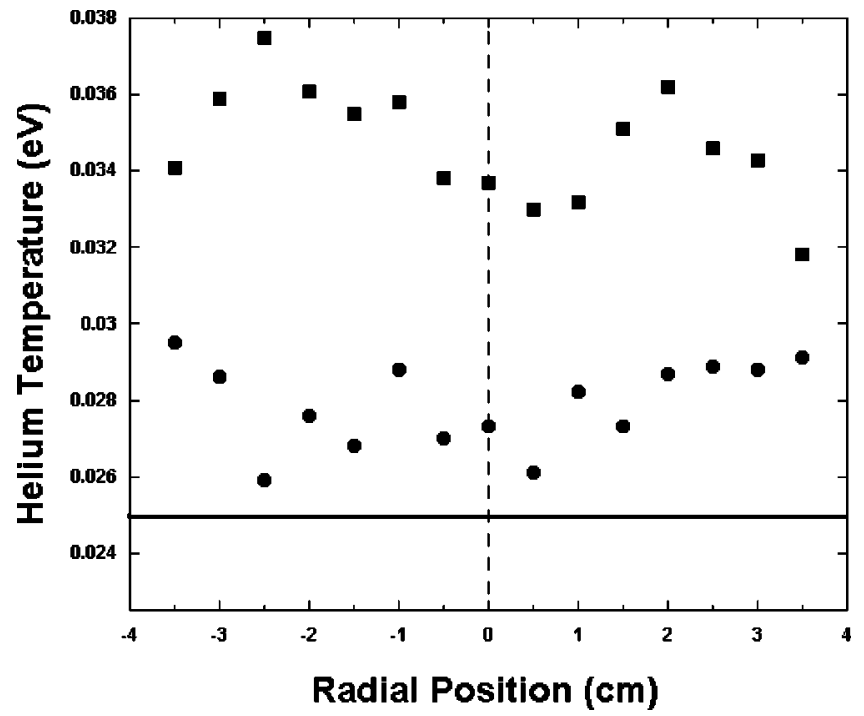

FIG. 8. He neutral temperature vs radius for two plasma conditions $(\bullet) B$ $=600 \mathrm{G}$ and $(\boldsymbol{\square}) B=1000 \mathrm{G}$. The hash vertical line corresponds to the center of the plasma column. The horizontal solid line indicates room temperature.

reaches $10^{12} \mathrm{~cm}^{-3}$. This large $2^{3} S$ to $2^{1} S$ population ratio results from the fact that no triplet states can directly decay to the singlet ground level of neutral helium. Meanwhile, all $N^{1} P$ singlet states $(N=1,2,3, \ldots)$ can quickly decay to the $1^{1} S$ ground state. Since all deexcitation toward the ground state must involve a $N^{1} P \rightarrow 1{ }^{1} S$ transition and that electronimpact excitation transfer cross sections for the $2^{1} S \rightarrow N^{1} P$ are about 30 times larger than the corresponding $2^{3} S$ $\rightarrow N^{1} P$ cross sections, ${ }^{54,55}$ the population of the $2^{3} S$ state will always be larger than the population of the $2^{1} S$ in helicon plasmas. Consequently, the nearby $2^{3} P$ state will also have large population when compared to the $2^{1} P$ population.

Second, the branching ratio of $3^{1} P$ to $2^{1} S$ is only 0.023 while it is unity for the triplet transition. This means that only $2.3 \%$ of the fluorescence radiation is emitted at 501.6 $\mathrm{nm}$ while $97.7 \%$ is radiated at $53.70 \mathrm{~nm}\left(3{ }^{1} P \rightarrow 1^{1} S\right)$. Finally, the PMT used in this experiment is roughly $30 \%$ more sensitive to 388.9 than $501.6 \mathrm{~nm}$ photons. ${ }^{33}$ Thus the diode laser LIF scheme is expected to yielded smaller $\mathrm{S} / \mathrm{N}$ ratio values and the $\mathrm{S} / \mathrm{N}$ ratio obtained in these experiments is close to optimal for the available apparatus. The neutral temperature of $0.027 \mathrm{eV}$ for the neutral velocity distribution function shown in Fig. 7 is very close to room temperature $(0.025 \mathrm{eV})$ and in all measurements performed for this work, the measured neutral temperature ranged from 0.025 to 0.05 $\mathrm{eV}$, consistent with previous dye laser LIF measurements. ${ }^{9}$ LIF neutral temperatures at even higher pressure (20-50 mTorr) are at or barely above room temperature (as expected), thus confirming the validity and precision of the LIF technique. We note that we were only able to complete He I LIF measurements at high neutral pressures ( $p_{\mathrm{He}}$ $>8$ mTorr), consistent with the hypothesis that frequent collisions are required to populate the $2^{1} P$ level.

Two radial scans of the helium plasma column are shown in Fig. 8. The neutral temperatures were measured every $0.5 \mathrm{~cm}$ under two different magnetic field conditions. 
All other source parameters remained the same as for Fig. 7 . The center of the column is identified as the zero position. In the $1000 \mathrm{G}$ case, the neutral temperature peaks off-axis at $r$ $\approx \pm 2.5 \mathrm{~cm}$. This position corresponds to the edge of the bright plasma column. Since heat is transferred to neutrals from ions via charge exchange and momentum transfer, the data appear to indicate that the ion heating is occuring near the edge of the plasma. Edge heating of ions in argon plasmas was recently proposed as evidence for the existence of slow waves at the edge of helicon sources. ${ }^{14}$ In this scenario, the ion heating waves (or slow waves) are heavily damped by the plasma and, consequently, heating occurs preferentially within the outskirts of the plasma column. Hot ions within the plasma edge encounter either free flowing neutral or magnetically confined cold ions from the core. Heat is transferred to the core by collision (ion-ion collisions) or transferred to the neutral (ion-neutral collisions). The ion temperature profile indicates a peak temperature at the outskirts with a flat ion temperature within the core. ${ }^{14,56,57}$ The neutral temperature profile indicates peak temperatures near the edge and cooler neutrals in the core and outside the column. The neutral helium temperature profile is also consistent with charge-exchange braking of cylindrical plasma rotation. Cylindrical rotation of argon helicon plasmas was observed in a series of recent two-dimensional dye laser LIF experiments in HELIX. ${ }^{58}$

The He I data suggest that neutral LIF could be used to investigate ion heating and plasma rotation in helicon plasma sources. In addition, the He I LIF intensities could be used to measure plasma density and neutral density profiles. However, the population of the initial LIF state $\left(2^{1} P\right)$ is a complex product of ground level electron-impact excitation and excitation transfer from neighboring levels, such as the metastable $2^{1} S$ state, ${ }^{50-52}$ and a collisional radiative model would be needed to relate plasma density to the population of the $2{ }^{1} P$ level ${ }^{50,51}$ Futhermore, the process of excitation transfer $\left(3^{1} D \rightarrow 3^{1} P\right)$ is also a complex function of plasma density and electron temperature ${ }^{54,55}$ and only a fraction of the excited $3{ }^{1} \mathrm{D}$ electrons will transfer to the $3{ }^{1} \mathrm{P}$ before the $3{ }^{1} \mathrm{D}$ state spontaneously decays. Thus, although density profile extraction from the LIF data is theoretically possible, development of such a diagnostic is unlikely because of its inherent complexity.

\section{ACKNOWLEDGMENTS}

This work was supported by the U.S. Department of Energy EPSCoR Laboratory Partnership Program under Grant No. ER45849. The authors are grateful to S. Cohen and B. Berlinger for their hospitality and technical assistance during the tests of the diode laser system on the MNX experiment at Princeton Plasma Physics Laboratory.

\footnotetext{
${ }^{1}$ R. A. Stern and J. A. Johnson III, Phys. Rev. Lett. 34, 1548 (1975).

${ }^{2}$ R. A. Stern, D. N. Hill, and N. Rynn, Phys. Lett. A 93, 127 (1983).

${ }^{3}$ D. N. Hill, S. Fornaca, and G. Wickman, Rev. Sci. Instrum. 54, 309 (1983).

${ }^{4}$ F. Anderegg et al., Phys. Rev. Lett. 57, 329 (1986)

${ }^{5}$ J. M. McChesney, R. A. Stern, and P. M. Bellan, Phys. Rev. Lett. 59, 1436 (1987).

${ }^{6}$ R. McWilliams and D. Sheehan, Phys. Rev. Lett. 56, 2485 (1986).
}

${ }^{7}$ D. A. Edrich, R. McWilliams, and N. S. Wolf, Rev. Sci. Instrum. 67, 2812 (1996).

${ }^{8}$ W. A. Noonan, T. G. Jones, and P. F. Ottinger, Rev. Sci. Instrum. 68, 1032 (1997).

${ }^{9}$ E. E. Scime et al., Plasma Sources Sci. Technol. 7, 186 (1998).

${ }^{10}$ J. L. Kline, E. E. Scime, P. A. Keiter, M. M. Balkey, and R. F. Boivin, Phys. Plasmas 6, 4767 (1999).

${ }^{11}$ P. A. Keiter, E. E. Scime, M. Balkey, R. F. Boivin, J. Kline, and P. Gary, Phys. Plasmas 7, 779 (2000).

${ }^{12}$ E. E. Scime, P. A. Keiter, M. M. Balkey, R. F. Boivin, J. L. Kline, M. Blackburn, and S. P. Gary, Phys. Plasmas 7, 2157 (2000).

${ }^{13}$ M. M. Balkey, R. F. Boivin, J. L. Kline, and E. E. Scime, Plasma Sources Sci. Technol. 10, 284 (2001).

${ }^{14}$ J. L. Kline et al., Phys. Rev. Lett. 88, 195002 (2002).

${ }^{15}$ G. D. Severn, D. A. Edrich, and R. McWilliams, Rev. Sci. Instrum. 69, 10 (1998)

${ }^{16}$ F. M. Levinton, Princeton Plasma Physics Laboratory PPPL (private communication).

${ }^{17}$ W. L. Wiese, M. W. Smith, and B. M. Glennon, Atomic Transitions Probabilities, Vol. 2 (National Standard Reference Data System NSRDS-NBS22, 1969).

${ }^{18}$ C. E. Moore, Atomic Energy Levels, Vol. 1 (National Standard Reference Data System NSRDS-NBS-1935, 1971).

${ }^{19}$ J. C. Camparo, Contemp. Phys. 26, 443 (1985).

${ }^{20}$ C. E. Weiman and L. Hollberg, Rev. Sci. Instrum. 62, 1 (1991).

${ }^{21}$ S. A Cohen, N. S. Siefert, S. Stange, R. F. Boivin, E. E. Scime, and F. M. Levinton, Phys. Plasmas 10, 1 (2003).

${ }^{22}$ MKS Instruments Inc., 6 Shattuck Rd., Andover, MA 01810-2449, 2000.

${ }^{23}$ P. A. Keiter, E. E. Scime, and M. M. Balkey, Phys. Plasmas 4, 2741 (1997).

${ }^{24}$ T. Y. Shoji et al., Plasma Sources Sci. Technol. 2, 5 (1993).

${ }^{25}$ I. D. Sudit and F. F. Chen, Plasma Sources Sci. Technol. 3, 162 (1994).

${ }^{26}$ M. G. Littman and H. J. Metcalf, Appl. Opt. 17, 2224 (1978).

${ }^{27}$ P. McNicholl and H. J. Metcalf, Appl. Opt. 24, 2757 (1985).

${ }^{28}$ Sacher Lasertechnik LLC, Instruction Manual, Hannah Arendt Str. 3-7 D35037 Marburg/Lahn, Germany, 2000.

${ }^{29}$ Burleigh Instruments, Burleigh Park, P.O. Box E, Fishers, NY 14453, 2000.

${ }^{30}$ National Instruments, 11500 N. Mopac Express Way, Austin, TX 78759, 2000.

${ }^{31}$ Oriel Instruments, 250 Long Beach Blvd., P.O. Box 872, Stratford, CT 06497, 2000.

${ }^{32}$ Dell Optics, 25 Bergen Blvd., Fairview, New Jersey 07022.

${ }^{33}$ Hamamatsu Corporation, Technical Datasheets, Box 6910, 360 Foothill Road, Bridgewater, NJ 08807, 2000.

${ }^{34}$ Stanford Research Systems Inc., 1290-D Reamwood Ave., Sunnyvale, CA 94089, 2000

${ }^{35}$ R. W. P. McWhirter, Plasma Diagnostics (Academic, New York, 1965), p. 201.

${ }^{36}$ R. L. Kurucz and B. Bell, Atomic Line Data, Kurucz CD-ROM No 23, Cambridge, MA, Smithsonian Astrophysical Observatory.

${ }^{37}$ A. Corney, Atomic and Laser Spectroscopy (Clarendon, Oxford, 1977).

${ }^{38}$ W. Demtröder, Laser Spectroscopy (Spinger-Verlag, Berlin, 1981).

${ }^{39}$ W. L. Wiese, in Plasma Diagnostic Techniques (Academic, New York, 1965), p. 265.

${ }^{40}$ H. R. Griem, Plasma Spectroscopy (McGraw-Hill, New York, 1964).

${ }^{41}$ H. R. Griem et al., Phys. Rev. 125, 177 (1962).

${ }^{42}$ H. R. Griem, Phys. Rev. 128, 515 (1962).

${ }^{43}$ M. Baranger, in Atomic and Molecular Processes (Academic, New York, 1962), p. 493.

${ }^{44}$ G. Marr, Plasma Spectroscopy (Elsevier, New York, 1968).

${ }^{45}$ S. Bashkin and J. O. Stoner, Atomic Energy Levels and Grotrian Diagrams (North Holland, Amsterdam, 1975).

${ }^{46}$ M. J. Goeckner and J. Goree, J. Vac. Sci. Technol. A 7, 977 (1989).

${ }^{47}$ R. F. Boivin, West Virginia University Plasma Physics Lab Report PL050, EPAPS-E-HPAEN-10-003306, 2003.

${ }^{48}$ J. McChesney, General Atomics Report GA-C22557, 1997.

${ }^{49}$ W. L. Wiese, M. W. Smith, and B. M. Glennon, Atomic Transitions Probabilities, Vol. 1 (National Standard Reference Data System NSRDSNBS-4, 1966).

${ }^{50}$ B. Brosda, Ph.D. thesis, Ruhr-Universitat, Bochum, 1993.

${ }^{51}$ See National Technical Information Service Document No. DE95502158INZ (S. Sasaki et al., He I Line Intensity Ratios in a Plasma 
for the Fusion Edge Plasmas NIFS-346, 1995). Copies may be ordered from the National Technical Information Service, Springfield, VA 22161.

${ }^{52}$ R. F. Boivin, J. L. Kline, and E. E. Scime, Phys. Plasmas 8, 5303 (2001).

${ }^{53}$ G. Herzberg, Atomic Spectra and Atomic Structure (Dover, New York, 1945).

${ }^{54}$ See National Technical Information Service Document No. DE96725192INZ (V. P. Shevelko and H. Tawara, Cross Sections for Electron-impact Induced Transitions between Excited States in He-n, n' $=2,3$ and 4, NIFS-DATA-28, 1995). Copies may be ordered from the
National Technical Information Service, Springfield, VA 22161.

${ }^{55}$ R. Denkelmann et al., J. Phys. B 32, 4635 (1999).

${ }^{56}$ J. L. Kline, M. M. Balkey, P. A. Keiter, E. E. Scime, A. M. Keesee, X. Sun, R. Hardin, C. Compton, R. F. Boivin, and M. W. Zintl, Phys. Plasmas 10, 2127 (2003).

${ }^{57}$ J. L. Kline, E. E. Scime, R. F. Boivin, A. M. Keesee, and X. Sun, Plasma Sources Sci. Technol. 11, 413 (2002).

${ }^{58}$ R. Hardin, C. Compton, A. Keesee, E. Scime, and X. Sun, Bull. Am. Phys. Soc. 47, BP1.103 (2002). 